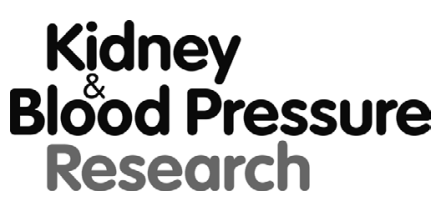

Kidney Blood Press Res 2018;43:1375-1387

DOI: $10.1159 / 000492952$

Published onlıne: 27 August, 2018

Accepted: 17 August, 2018

This article is licensed under the Creative Commons Attribution-NonCommercial-NoDerivatives 4.0 International License (CC BY-NC-ND) (http//www.kargercom/Services/OpenAccessLicense). Usage and distribution for commercial purposes as well as any distribution of modified material requires written permission.

\title{
Central Aortic Systolic Blood Pressure Exhibits Advantages Over Brachial Blood Pressure Measurements in Chronic Kidney Disease Risk Prediction in Women
}

\author{
Linfeng Zhang Zengwu Wang Zuo Chen Xin Wang Ye Tian Lan Shao \\ Manlu Zhu \\ Division of Prevention and Community Health, National Center for Cardiovascular Disease, Fuwai \\ Hospital, Peking Union Medical College and Chinese Academy of Medical Science, Beijing, China
}

\section{Key Words}

Central systolic blood pressure $\cdot$ Chronic kidney disease $\cdot$ Epidemiology

\begin{abstract}
Background/Aims: To investigate whether the invasively obtained central aortic systolic blood pressure (CSBP) predicts chronic kidney disease (CKD) better than brachial systolic blood pressure (SBP), brachial diastolic blood pressure (DBP) and brachial pulse pressure (PP) in the middle-aged Chinese population. Methods: A cross-sectional study was carried out across China in 2009-2010 among the subjects aged 35-64 years. CSBP was measured noninvasively by radial artery applanation tonometry B-pro (A-PULSE CASP and corresponding software). CSBP, SBP, DBP and PP were standardized with Z-score and the odds ratios were calculated with multivariable logistic regression model. Results: Data of 10197 participants were analyzed. The multivariable logistic regression after adjusting for possible confounders showed that a 1-standard deviation increment in each blood pressure measurement was associated with greater risk of CKD in both men and women $(P<0.05)$. The association of CSBP with CKD was stronger than SBP, DBP and PP in women, while in men the association of CSBP with CKD was stronger only than PP. With CSBP and SBP entering into the multivariable logistic regression models jointly, the odds ratio (95\% confidence interval) for CSBP and SBP was 1.57 (1.39-1.79) and 1.22 (1.07-1.38) in women and 1.20 (1.03-1.39) and 1.48 (1.28-1.72) in men, respectively. With CSBP and DBP entering into the multivariable logistic regression models jointly, the odds ratio (95\% confidence interval) for CSBP and DBP was 1.68 (1.52-1.84) and 1.15 (1.04-1.27) in women and 1.30 (1.15-1.46) and 1.45 (1.29-1.63) in men, respectively. With CSBP and PP entering into the multivariable logistic regression models jointly, the odds ratio (95\% confidence interval) for CSBP and PP was 1.75 (1.58-1.94) and 1.06 (0.96-1.17) in women and 1.58 (1.41-1.77) and 1.04 (0.93-1.17) in men, respectively. Conclusion: CSBP and
\end{abstract}




\section{Kidney Blood Pressure Research}

brachial blood pressure measurements are all predictors of CKD, however the non-invasively obtained CSBP may offer advantages over brachial blood pressure measurements in CKD risk prediction in women.

\section{Introduction}

Hypertension is the leading factor in the global burden of disease [1]. It is the number one risk for mortality because of its dominant role in cardiovascular pathogenesis. Effective control of hypertension can reduce the risk of coronary artery disease, stroke, peripheral vascular disease, vision loss, heart failure, and renal failure [2]. Therefore, accurate assessment and effective treatment for hypertension are absolutely essential.

Although mean and diastolic arterial pressure remain similar in all large arteries [3], systolic blood pressure varies throughout the arterial tree [4] and systolic pressure is significantly higher in the brachial artery than in the aorta. Brachial blood pressure measurements were introduced into medical practice over 100 years and remain the principal tool used for the clinical diagnosis and monitoring of hypertension. However, emerging evidences now suggest central blood pressure may be a better predictor of cardiovascular prognosis than the brachial blood pressure. Studies showed that central systolic blood pressure (CSBP) is more relevant to cardiovascular disease [5,6]; moreover, anti-hypertensive drugs can exert differential effects on brachial and central pressure and evaluation of the antihypertensive effect based on CSBP may be better than brachial blood pressure [7]. However, the current evidences are still scant.

On the other hand, although intravascular catheterization is still the gold standard for central blood pressure assessment, recent progress in medical technologies has enabled non-invasive assessment of central blood pressure, which is more suitable for use in routine clinical practice. Kidney is the important organ in the middle of the abdominal aorta and is exposed to the pressure at the level of renal arteries, which is correlated more closely with central blood pressure rather than brachial blood pressure. Studies have indicated that aortic stiffness or the invasively obtained pulse pressure in the ascending aorta are related to proteinuria or reduced renal function $[8,9]$. However, whether the CSBP obtained with invasive devices is a better marker of risk for CKD than traditional brachial blood pressure measurements has been poorly studied. To answer this question we compared CSBP obtained using the non-invasive device with the traditional brachial blood pressure measurements in relation to chronic kidney disease prevalence in a large scale middle-aged Chinese population in this study.

\section{Materials and Methods}

\section{Ethics approval and consent to participate}

Written informed consent was obtained from all participants and institutional review board approval was obtained from the Fuwai Hospital.

\section{Study design and participants}

A cross-sectional survey on cardiovascular disease and related risk factors was conducted in 20092010 across China. The details of this survey have been described previously [10-13]. The participants came from 12 subpopulations, including subpopulations that came from Beijing, Heilongjiang province, Shanxi province, Shaanxi province, Sichuan province, Jiangsu Province, Zhejiang province, Yunnan province, Guangdong province, Inner Mongolia Autonomous Region, Xinjiang Uygur Autonomous Region, and Tibet Autonomous Region. The subpopulations were selected in consideration of economic and social development and located in different parts of China. The sampling method in our survey referred to in the China Multicenter Collaborative Study of Cardiovascular Epidemiology was initiated in 1982 [14, 15]. 


\section{Kidney Blood Pressure Research}

Briefly, each subpopulation consisted of about 1000 men and women aged 35-64 years, randomly selected in clusters (villages or residential households). Written informed consent was obtained from all participants and institutional review board approval was obtained from the Fuwai Hospital.

For this analysis, we excluded those participants with missing data for the variables analyzed; only the data of the participants with complete data were analyzed.

\section{Data collection and measurements}

The demographic, personal habits such as cigarette smoking and alcohol consumption, personal and family history of cardiovascular disease, and physical examination data were collected by trained research staffs per the study protocol. Smoking status was classified into two categories: current smokers and nonsmokers. Alcohol consumption was classified into two categories: current drinkers and non-drinkers. Blood pressure was measured three times using a mercury sphygmomanometer with the participant in the sitting position after 5 minutes of rest. Systolic blood pressure (SBP) and diastolic blood pressure (DBP) were recorded corresponding to the appearance of the first and fifth Korotkoff phase sounds, respectively. The average of the three measurements was used for analysis. CSBP was obtained using the BPro device with A-Pulse CASP software (Health STATS, Singapore) [16 - 18]. The radial artery waveform was captured first, and then waveforms were averaged from single waveforms recorded consecutively for 10 seconds per block of waveforms. From the radial pulse wave, the software estimates the CSBP using an n-point moving average method, a mathematical low-pass filter [19]. Studies have shown an accurate agreement in CSBP compared with invasively measured CSBP $[16,17]$. BMI was calculated using the formula weight $/ \mathrm{height}^{2}\left(\mathrm{~kg} / \mathrm{m}^{2}\right)$. If a subject's parents or siblings have been reported to have the history of hypertension, diabetes mellitus, stroke and coronary heart disease, the subject was considered to have family history of cardiovascular disease (CVD). If a subject has suffered from the disease of a kidney, such as nephritis, nephrolithiasis, hydronephrosis, etc, the subject was considered to have history of kidney disease.

All blood samples were obtained in the morning after at least 8-h overnight fast, and biochemical assays were performed at a central laboratory. The serum glucose (GLU) was determined using enzymatic method. The serum total cholesterol (TC), high-density lipoprotein cholesterol (HDL-C), and triglyceride (TG) were determined using enzymatic methods with an autoanalyzer (HITACHI 7080, Japan). Highsensitivity C-reactive protein (hsCRP) was measured by latex agglutination immunoturbidimetry assay. Uric acid was measured by enzymatic method. Urinary microalbumin was measured using radioimmunoassay method. Urinary creatinine and serum creatinine were measured by enzymatic method. Urinary albumin to creatinine ratio (ACR) was calculated. The estimated glomerular filtration rate (eGFR) was calculated using the CKD-EPI equation, $\left\{\mathrm{eGFR}\left(\mathrm{mL} / \mathrm{min}\right.\right.$ per $\left.1.73 \mathrm{~m}^{2}\right)=141 \times \min (\mathrm{SCr} / \kappa, 1)^{\alpha} \times \max (\mathrm{SCr} / \kappa, 1)^{-1.209} \times 0.993^{\text {Age }}$ $\times 1.018$ [if female] $\times 1.159$ [if African American], where SCr is serum creatinine, $\kappa$ is 0.7 for females and 0.9 for males, $\alpha$ is -0.329 for females and -0.411 for males, min indicates the minimum of $\mathrm{SCr} / \kappa$ or 1 , and max indicates the maximum of SCr/K or 1\} [20]. CKD was defined as either decreased eGFR (eGFR $<60 \mathrm{~mL}$ / $\mathrm{min} / 1.73 \mathrm{~m}^{2}$ ) or albuminuria (ACR $\geq 30 \mathrm{mg} / \mathrm{g}$ ) according to Kidney Disease Improving Global Outcomes (KDIGO) guidelines [21].

\section{Statistical analysis}

Continuous variables were presented as mean and standard deviation for the data that were approximately normally distributed and median and interquartile range for the data that were not normally distributed and compared with $\mathrm{t}$ test or Wilcoxon signed-rank test where appropriate; Categorical variables were presented as frequencies and percentages and compared with chi-square test. To explore the correlation of different blood pressure measurements with eGFR and ACR, the spearman correlation coefficients were calculated. To compare the association of different blood pressure measurements with CKD, receiver operating characteristics (ROC) analyses were employed and the area under the ROC curve (AUC) of the blood pressure measurements was calculated and the univariable and multivariable logistic regression analysis based on Z-score standardization was performed. The association of a factor with CKD was analyzed and the factor showing a $P$ value $<0.05$ was entered into the multivariable logistic regression model as the covariate. Before conducting the multivariable regression, a diagnosis for multicollinearity among covariates was assessed. 


\section{Kidney Bloód Pressure Research}

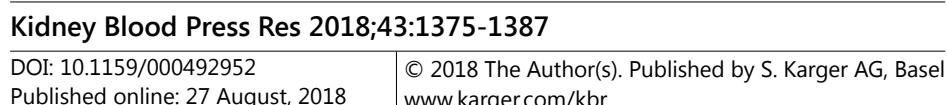

Published online: 27 August, 2018 www.karger.com/kb

All the analyses were carried out using SAS version 9.4 (SAS institute, Cary, NC, USA). All $P$ values were 2 sided and values $<0.05$ were considered significant.

\section{Results}

Demographic and clinical data of the subjects analyzed

Of the 14046 individuals invited, 11623 participated in the survey and the data of 10197 participants were analyzed. Table 1 summarized the characteristics of participants analyzed. Of the 10197 participants, 4752 were men and 5445 were women. The mean age was 50.0 (8.0) years. Men had a higher level of SBP, DBP, TG, GLU, uric acid and HsCRP and a lower level of BMI, TC, HDL-C, eGFR and ACR. The proportion of participants with smoking, alcohol consumption, education status above high school, history of myocardial infarction,

Table 1. Characteristics of the study participants. Data are presented as mean (standard deviation), median (interquartile range), or frequencies (percentages). The $P$ value given is for the test, Wilcoxon test or the $\chi^{2}$ test. Abbreviations: CKD, chronic kidney disease; BMI, body mass index; CSBP, central systolic blood pressure; SBP, systolic blood pressure; DBP, Diastolic blood pressure; PP, pulse pressure; TC, total cholesterol; HDL-C, high-density lipoprotein cholesterol; TG, triglycerides; GLU, fasting blood glucose; eGFR, estimated glomerular filtration rate; ACR, albumin to creatinine ratio; hsCRP, high-sensitivity C-reactive protein. a, $P$ value for the difference between men and women; $b, P$ value for the difference between the participants with and without CKD

\begin{tabular}{|c|c|c|c|c|c|c|}
\hline Parameters & $\begin{array}{c}\text { Men } \\
(\mathrm{n}=4752)\end{array}$ & $\begin{array}{l}\text { Women } \\
(\mathrm{n}=5445)\end{array}$ & $\begin{array}{c}P- \\
\text { value }^{\mathrm{a}}\end{array}$ & $\begin{array}{l}\text { Without CKD } \\
\quad(\mathrm{n}=8842)\end{array}$ & $\begin{array}{c}\text { With } \\
\text { CKD } \\
(\mathrm{n}=1355)\end{array}$ & $\begin{array}{c}P- \\
\text { value }^{\mathrm{b}}\end{array}$ \\
\hline Age (years) & $50.1(8.2)$ & $50.0(7.9)$ & 0.536 & $49.8(8.0)$ & $51.7(7.9)$ & $<0.001$ \\
\hline BMI $\left(\mathrm{kg} / \mathrm{m}^{2}\right)$ & $24.4(3.5)$ & $24.7(3.7)$ & $<0.001$ & $24.4(3.5)$ & $25.5(4.0)$ & $<0.001$ \\
\hline CSBP (mmHg) & $120.4(17.8)$ & $120.5(19.5)$ & 0.815 & $118.8(17.4)$ & $131.5(22.9)$ & $<0.001$ \\
\hline SBP (mmHg) & $130.2(19.6)$ & $128(20.8)$ & $<0.001$ & $127.2(18.8)$ & $141(24.8)$ & $<0.001$ \\
\hline $\mathrm{DBP}(\mathrm{mmHg})$ & $84.1(11.6)$ & $81.1(11.1)$ & $<0.001$ & $81.7(10.9)$ & $88(13.4)$ & $<0.001$ \\
\hline PP (mmHg) & $46.1(14.1)$ & $46.9(14.3)$ & 0.006 & $45.6(13.4)$ & $53(17.6)$ & $<0.001$ \\
\hline $\mathrm{TC}(\mathrm{mmol} / \mathrm{l})$ & $4.82(0.92)$ & $4.90(0.94)$ & $<0.001$ & $4.84(0.93)$ & $4.97(0.96)$ & $<0.001$ \\
\hline HDL-C (mmol/l) & $1.31(0.30)$ & $1.42(0.30)$ & $<0.001$ & $1.37(0.3)$ & $1.37(0.31)$ & 0.518 \\
\hline $\mathrm{TG}(\mathrm{mmol} / \mathrm{l})$ & $\begin{array}{l}1.32(0.92 \\
2.02)\end{array}$ & $\begin{array}{l}1.23(0.89 \\
1.81)\end{array}$ & $<0.001$ & $\begin{array}{l}1.56(0.89 \\
1.85)\end{array}$ & $\begin{array}{l}1.83(1.01 \\
2.27)\end{array}$ & $<0.001$ \\
\hline GLU (mmol/l) & $5.81(1.52)$ & $5.72(1.44)$ & 0.002 & $5.67(1.29)$ & $6.32(2.29)$ & $<0.001$ \\
\hline Current smoker, n (\%) & $2678(56.4)$ & $370(6.8)$ & $<0.001$ & $2722(30.8)$ & $326(24.1)$ & $<0.001$ \\
\hline Current drinker, n (\%) & $1683(35.4)$ & $200(3.7)$ & $<0.001$ & $1654(18.7)$ & $229(16.9)$ & 0.111 \\
\hline $\begin{array}{l}\text { High school education or higher, } \mathrm{n} \\
(\%)\end{array}$ & $1103(23.2)$ & 933(17.1) & $<0.001$ & $1794(20.3)$ & $242(17.9)$ & 0.037 \\
\hline eGFR $\left(\mathrm{ml} / \mathrm{min}\right.$ per $\left.1.73 \mathrm{~m}^{2}\right)$ & $98.8(12.4)$ & $100.8(12.4)$ & $<0.001$ & $100.4(11.4)$ & $96.5(17.4)$ & $<0.001$ \\
\hline ACR (mg/g) & $6.8(3.7,13.4)$ & $9.5(5.1,19.5)$ & $<0.001$ & $8.8(4.0,12.0)$ & $\begin{array}{l}81.5(37.4 \\
88.0)\end{array}$ & $<0.001$ \\
\hline History of kidney disease, n (\%) & $160(3.4)$ & $201(3.7)$ & 0.376 & $292(3.3)$ & $69(5.1)$ & $<0.001$ \\
\hline $\begin{array}{l}\text { History of myocardial infarction, } \mathrm{n} \\
(\%)\end{array}$ & $15(0.3)$ & $6(0.1)$ & 0.022 & $16(0.2)$ & $5(0.4)$ & 0.155 \\
\hline History of stroke, n (\%) & $107(2.3)$ & $47(0.9)$ & $<0.001$ & $117(1.3)$ & $37(2.7)$ & $<0.001$ \\
\hline $\begin{array}{l}\text { Family history of cardiovascular } \\
\text { disease, n (\%) }\end{array}$ & $2726(57.4)$ & $3260(59.9)$ & 0.010 & $5071(57.4)$ & $915(67.5)$ & $<0.001$ \\
\hline Uric acid $(\mu \mathrm{mol} / \mathrm{l})$ & $312(77)$ & $234(63)$ & $<0.001$ & $270(80)$ & $271(86)$ & 0.568 \\
\hline $\operatorname{HsCRP}(\mathrm{mg} / \mathrm{l})$ & $0.9(0.5,1.9)$ & $0.8(0.4,1.8)$ & $<0.001$ & $1.9(0.4,1.8)$ & $2.6(0.6,2.4)$ & $<0.001$ \\
\hline North region, n (\%) & $2646(55.7)$ & $3130(57.5)$ & 0.067 & $4890(55.3)$ & $886(65.4)$ & $<0.001$ \\
\hline Urban area, n (\%) & $1491(31.4)$ & $1642(30.2)$ & 0.183 & $2699(30.5)$ & $434(32.0)$ & 0.264 \\
\hline
\end{tabular}




\section{Kidney \\ Blood Pressure Research}

and history of stroke was higher in men than in women. The proportion of participants with family history of cardiovascular disease was lower in men than in women. Age and CSBP levels and the proportion of the participants with history of kidney disease, and the proportion of the participants living the north region and urban area were similar in both genders. With eGFR $<60 \mathrm{~mL} / \mathrm{min} / 1.73 \mathrm{~m}^{2}$ and ACR $\geq 30 \mathrm{mg} / \mathrm{g}$ as the indicator of CKD, there were a total of 1355 participants that were diagnosed as CKD and the overall prevalence of CKD was $13.3 \%$. There were 40 participants with eGFR $<60 \mathrm{~mL} / \mathrm{min} / 1.73 \mathrm{~m}^{2}$ and 487 participants with ACR $\geq 30 \mathrm{mg} / \mathrm{g}$ in men and 34 participants with eGFR $<60 \mathrm{~mL} / \mathrm{min} / 1.73 \mathrm{~m}^{2}$ and 837 participants with ACR $\geq 30 \mathrm{mg} / \mathrm{g}$ in women. The prevalence of CKD was $10.7 \%$ in men and $15.6 \%$ in women, respectively. The participants with CKD were older and had a higher level of BMI, CSBP, SBP, DBP, PP, TC, TG, GLU, ACR and HsCRP than the participants without CKD (Table 1). The proportion of participants with history of kidney disease, history of stroke, family history of cardiovascular disease and living in the north region was higher in the participants with CKD, while the proportion of participants with smoking, education status above high school was lower in the participants without CKD.

\section{Correlation of different blood pressure measurements with eGFR and ACR}

Table 2 shows the spearman correlation coefficient for different blood pressure measurements with eGFR and ACR by age and gender. The correlation of the indices with eGFR in the gender- and age-groups was weak and most of the correlation coefficients were less than 0.1. The correlation of the indices with ACR in the gender- and age-groups was stronger and all correlation coefficients were statistically significant $(P<0.001)$. In men, the strongest correlation was observed between SBP and ACR in the overall age group, whereas CSBP had the highest correlation coefficient with ACR in 45-54 year group. In women, the strongest correlation was observed between CSBP and ACR in the overall and all age-specific groups.

\section{ROC curve analysis of different blood pressure measurements for identifying CKD}

Fig. 1 and 2 presented the ROC curve of each blood pressure measurement for identifying CKD by age in men and women, respectively. SBP showed the highest AUC in men and CSBP showed highest AUC in women in the overall age group. For specific sex and age group, DBP

Table 2. Spearman correlation coefficients of blood pressure measurements with eGFR and ACR by gender and age. Symbols denote significant differences $\left({ }^{*} P<0.05\right.$; ${ }^{\sharp} P<0.01$; $\left.{ }^{\S} P<0.001\right)$ within each age and sex group. Abbreviations: eGFR, estimated glomerular filtration rate; ACR, albumin to creatinine ratio; CSBP, central aortic systolic blood pressure; SBP, systolic blood pressure; DBP, diastolic blood pressure; PP, pulse pressure

\begin{tabular}{lcccccccc}
\hline & \multicolumn{3}{c}{ eGFR } & \multicolumn{5}{c}{ ACR } \\
& $35-44$ & $45-54$ & $55-64$ & total & $35-44$ & $45-54$ & $55-64$ & total \\
\hline Men & & & & & & & & \\
N & 1374 & 1767 & 1611 & 4752 & 1374 & 1767 & 1611 & 4752 \\
CSBP & 0.010 & -0.038 & $-0.059^{*}$ & $-0.166 \S$ & $0.229 \S$ & $0.271 \S$ & $0.224 \S$ & $0.266 \S$ \\
SBP & 0.003 & -0.020 & $-0.090 \S$ & $-0.179 \S$ & $0.236 \S$ & $0.259 \S$ & $0.266 \S$ & $0.281 \S$ \\
DBP & $-0.078^{\#}$ & $-0.089 \S$ & $-0.101 \S$ & $-0.124 \S$ & $0.194 \S$ & $0.238 \S$ & $0.170 \S$ & $0.218^{\S}$ \\
PP & $0.075^{\#}$ & $0.061^{*}$ & -0.039 & $-0.137 \S$ & $0.129 \S$ & $0.158 \S$ & $0.222 \S$ & $0.198 \S$ \\
Women & & & & & & & & \\
N & 1544 & 2099 & 1802 & 5445 & 1545 & 2099 & 1802 & 5445 \\
CSBP & -0.042 & $0.047^{*}$ & 0.003 & $-0.190 \S$ & $0.193 \S$ & $0.277 \S$ & $0.280 \S$ & $0.282 \S$ \\
SBP & -0.036 & 0.027 & -0.003 & $-0.211 \S$ & $0.171 \S$ & $0.259 \S$ & $0.250 \S$ & $0.265 \S$ \\
DBP & $-0.060^{*}$ & $-0.049^{*}$ & -0.030 & $-0.141 \S$ & $0.152 \S$ & $0.228 \S$ & $0.182 \S$ & $0.212^{\S}$ \\
PP & $<-0.001$ & $0.082 \S$ & 0.024 & $-0.187 \S$ & $0.117 \S$ & $0.189 \S$ & $0.201 \S$ & $0.211 \S$ \\
\hline
\end{tabular}




\section{Kidney \\ Blood Pressure \\ Research}

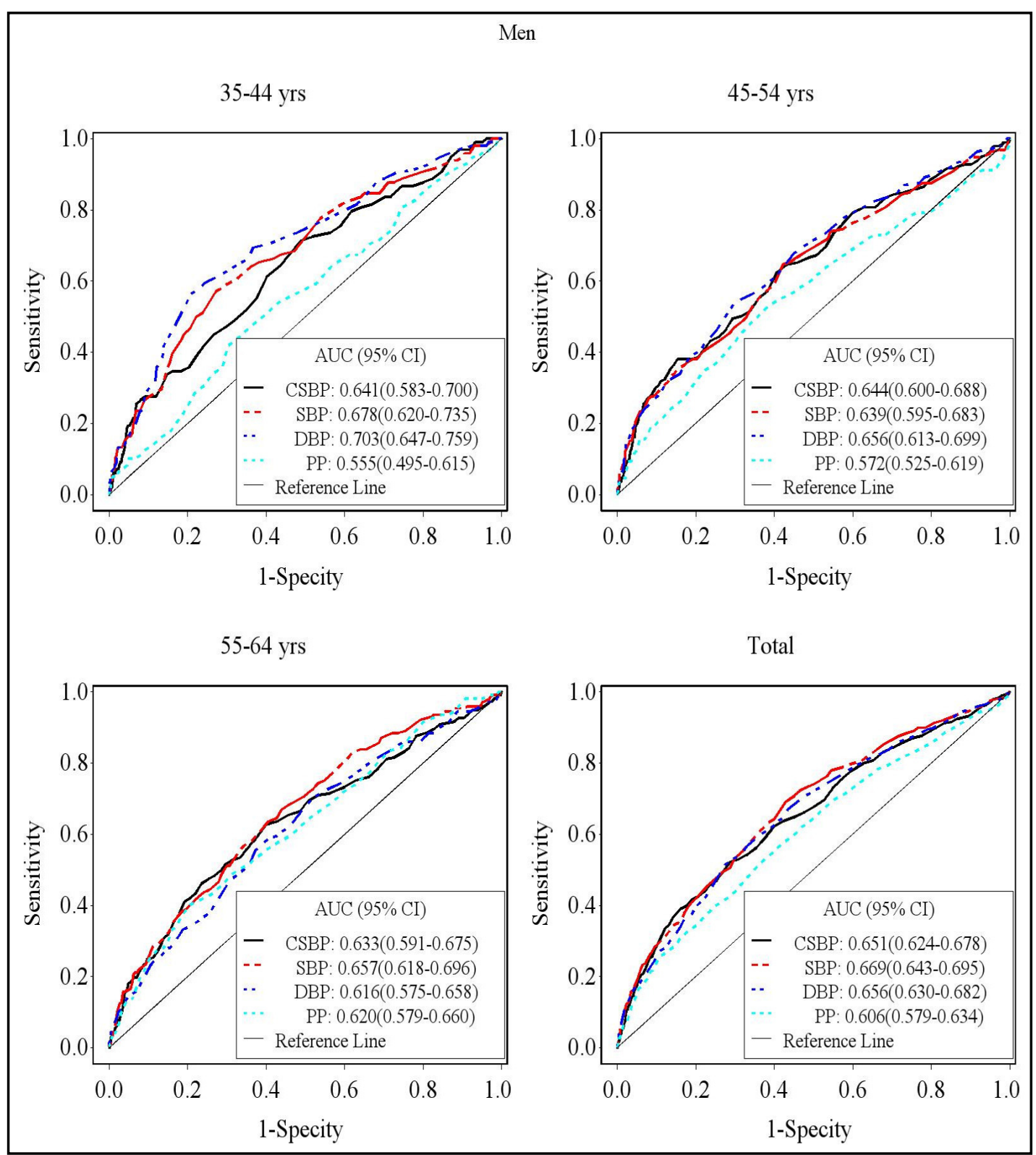

Fig. 1. ROC curves of different blood pressure measurements to identify CKD by age group in women. Abbreviations: ROC, receiver operating characteristics; CKD, chronic kidney disease; AUC, area under the curve; CI, confidence interval; CSBP, central aortic systolic blood pressure; SBP, systolic blood pressure; DBP, diastolic blood pressure; PP, pulse pressure.

had the highest AUC in 35-44 year and 45-54 year group and SBP had the highest AUC in 55-64 year group in men, SBP had the highest AUC in 35-44 year group and CSBP had the highest AUC in 45-54 year and 55-65 year group in women.

\section{Logistic analysis of different blood pressure measurements with CKD}

Comparison of odds ratios for CKD associated with different blood pressure measurements based on Z-score standardization by age and gender was exhibited in Table 3. As shown, a 1-SD increment in each blood pressure index was associated with greater risk of CKD in gender- and age-specific group (all $P<0.05$ ). In men, SBP was more strongly associated with CKD than other blood pressure measurements before and after adjusting 


\section{Kidney \\ Bloód Pressure Research}

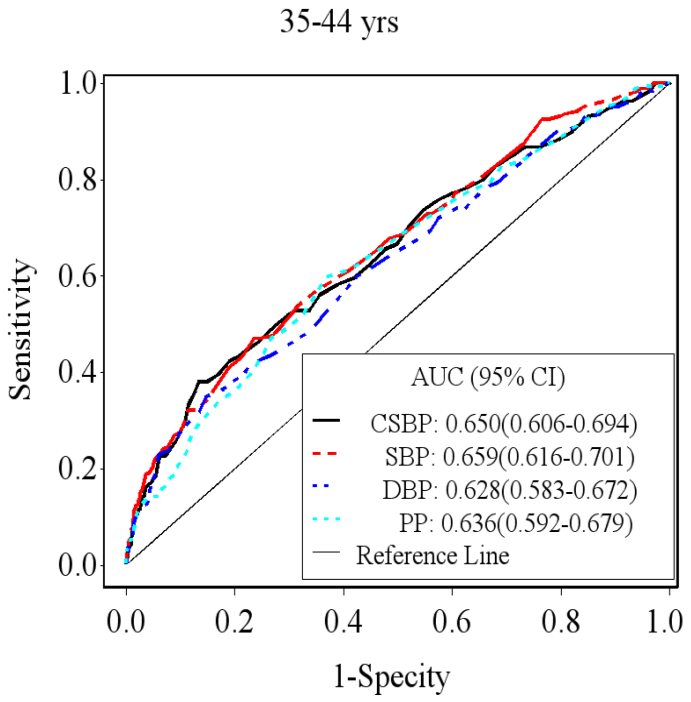

$55-64$ yrs

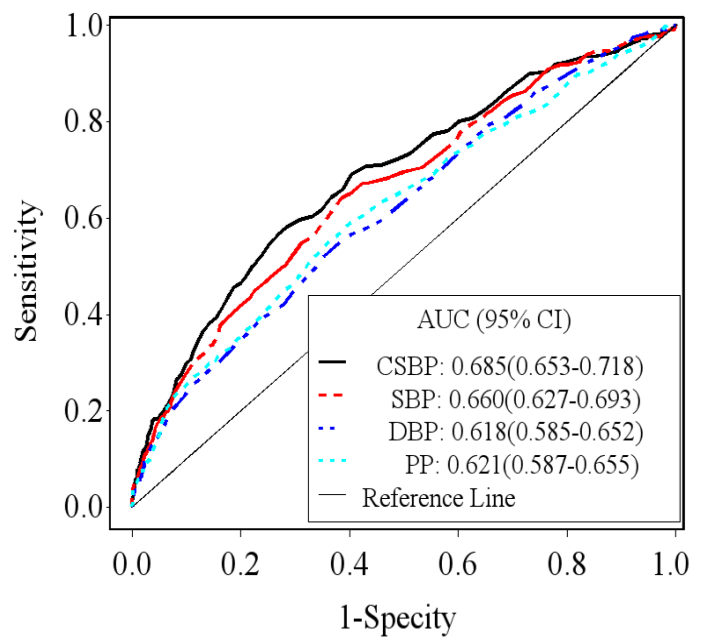

$45-54$ yrs

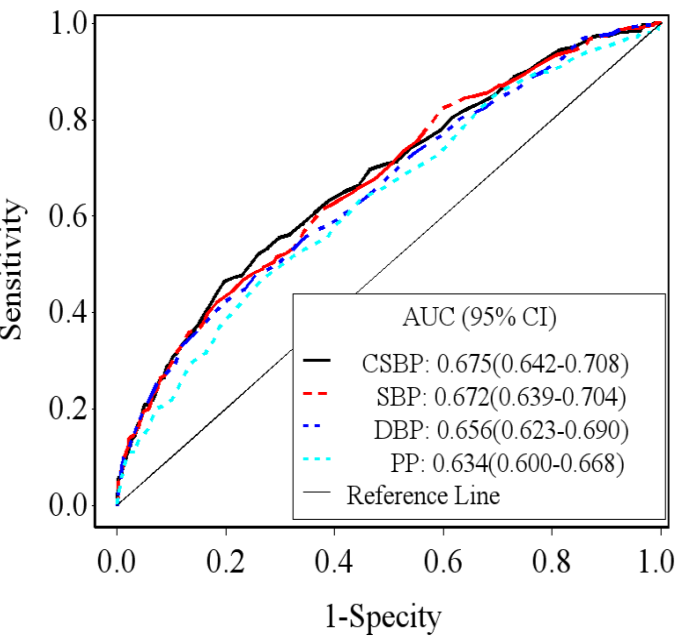

Total

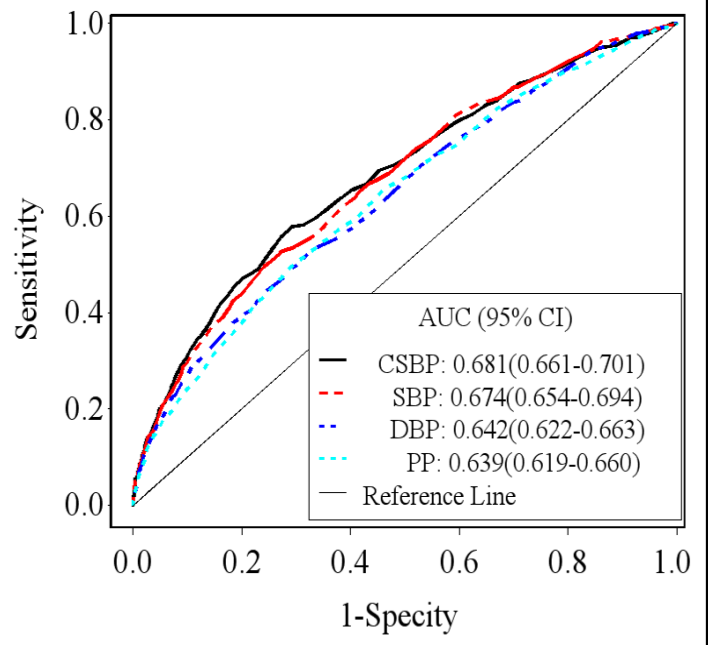

Fig. 2. ROC curves of different blood pressure measurements to identify CKD by age group in women. Abbreviations: ROC, receiver operating characteristics; CKD, chronic kidney disease; AUC, area under the curve; CI, confidence interval; CSBP, central aortic systolic blood pressure; SBP, systolic blood pressure; DBP, diastolic blood pressure; PP, pulse pressure.

for the potential confounding factors in the overall age group; for specific age group the measurement with the best performance in identifying CKD was DBP in 35-44 year and 4554 year group and SBP in 55-64 year group. In women, CSBP was more strongly associated with CKD than other blood pressure measurements before and after adjusting for other factors in the overall age group; for the specific age group the measurement with the best performance in identifying CKD was SBP in 35-44 year group and CSBP in 45-54 year and 55-64 year group. PP consistently showed the weakest association with CKD in both men and women and in each age group.

The multicollinearity diagnosis revealed that CSBP and the brachial blood pressure parameters had a value of variance inflation less than 4 , therefore to compare the strength of the associations, CSBP and another brachial blood pressure variable were entered into 


\section{Kidney Blood Pressure Research}

Table 3. OR and 95\% CI to identify CKD of different blood pressure measurements by gender and age groups. OR and 95\% CI were obtained with the logistic regression models. The blood pressure measurements were standardized with z score. *, Adjusted for age, smoking, body mass index (BMI), total cholesterol, fasting glucose, triglyceride, high-sensitivity C reaction protein (hsCRP), history of kidney disease, history of stroke, family history of cardiovascular disease, education level, north or south regions. The blood pressure measurements were standardized with $\mathrm{Z}$ score. Abbreviations: OR, Odds ratio; $\mathrm{CI}$, confidence interval; CKD, chronic kidney disease; CSBP, central aortic systolic blood pressure; SBP, systolic blood pressure; DBP, diastolic blood pressure; PP, pulse pressure

\begin{tabular}{|c|c|c|c|c|c|}
\hline & & \multicolumn{4}{|c|}{ Age groups(yrs) } \\
\hline & & $35-44$ & $45-54$ & $55-64$ & Total \\
\hline \multicolumn{6}{|l|}{ Men } \\
\hline \multirow[t]{2}{*}{ CSBP Z-score } & Crude OR & $1.64(1.36-1.97)$ & $1.67(1.46-1.92)$ & $1.60(1.40-1.84)$ & $1.70(1.56-1.85)$ \\
\hline & Adjusted OR* & $1.47(1.19-1.82)$ & $1.71(1.48-1.98)$ & $1.59(1.38-1.85)$ & $1.62(1.47-1.78)$ \\
\hline \multirow[t]{2}{*}{ SBP Z-score } & Crude OR & $1.81(1.51-2.16)$ & $1.70(1.48-1.95)$ & $1.74(1.52-2.00)$ & $1.80(1.66-1.96)$ \\
\hline & Adjusted OR* & $1.66(1.35-2.03)$ & $1.73(1.49-2.00)$ & $1.70(1.46-1.97)$ & $1.71(1.56-1.88)$ \\
\hline \multirow[t]{2}{*}{ DBP Z-score } & Crude OR & $2.02(1.67-2.44)$ & $1.79(1.55-2.06)$ & $1.54(1.34-1.77)$ & $1.76(1.61-1.92)$ \\
\hline & Adjusted $\mathrm{OR}^{*}$ & $1.84(1.50-2.27)$ & $1.85(1.59-2.15)$ & $1.48(1.27-1.72)$ & $1.69(1.54-1.85)$ \\
\hline \multirow[t]{2}{*}{ PP Z-score } & Crude OR & $1.24(1.02-1.51)$ & $1.32(1.14-1.51)$ & $1.53(1.34-1.75)$ & $1.47(1.35-1.60)$ \\
\hline & Adjusted OR* & $1.11(0.89-1.37)$ & $1.28(1.10-1.48)$ & $1.50(1.30-1.73)$ & $1.35(1.23-1.48)$ \\
\hline \multicolumn{6}{|l|}{ Women } \\
\hline \multirow[t]{2}{*}{ CSBP Z-score } & Crude OR & $1.74(1.52-2.00)$ & $1.91(1.71-2.15)$ & $1.97(1.75-2.22)$ & $1.94(1.81-2.09)$ \\
\hline & Adjusted OR* & $1.67(1.42-1.95)$ & $1.79(1.58-2.04)$ & $1.87(1.65-2.12)$ & $1.81(1.67-1.97)$ \\
\hline \multirow[t]{2}{*}{ SBP Z-score } & Crude OR & $1.81(1.57-2.07)$ & $1.89(1.68-2.11)$ & $1.82(1.61-2.04)$ & $1.89(1.76-2.03)$ \\
\hline & Adjusted OR* & $1.71(1.46-2.01)$ & $1.73(1.52-1.97)$ & $1.68(1.48-1.90)$ & $1.73(1.59-1.87)$ \\
\hline \multirow[t]{2}{*}{ DBP Z-score } & Crude OR & $1.66(1.43-1.91)$ & $1.83(1.62-2.06)$ & $1.59(1.41-1.78)$ & $1.73(1.61-1.86)$ \\
\hline & Adjusted OR* & $1.56(1.32-1.83)$ & $1.65(1.45-1.87)$ & $1.51(1.34-1.72)$ & $1.55(1.44-1.68)$ \\
\hline \multirow[t]{2}{*}{ PP Z-score } & Crude OR & $1.64(1.42-1.89)$ & $1.62(1.45-1.82)$ & $1.60(1.43-1.79)$ & $1.66(1.55-1.78)$ \\
\hline & Adjusted OR* & $1.49(1.28-1.74)$ & $1.48(1.31-1.67)$ & $1.46(1.29-1.64)$ & $1.49(1.38-1.62)$ \\
\hline
\end{tabular}

the multivariable logistic regression models jointly and the odds ratios were compared. The results showed that in the overall age group all blood pressure variables were independent predictor of CKD prevalence in both men and women except PP (Table 4). In women, the association of CSBP with CKD was stronger than SBP, DBP and PP (the 95\% confidence interval did not overlap with each other for CSBP and SBP, CSBP and DBP, CSBP and PP), while in men the association of CSBP with CKD was stronger only than PP and there were no statistically significant difference between the odds ratio of CSBP and SBP, CSBP and DBP (the 95\% confidence interval overlapped with each other). In women, after adjusted for the possible confounding factors and another blood pressure variable, CSBP was consistently the significant independent risk factor of CKD in each age group except the 35-44 age group of model 1. Although there was no statistical significance, the data indicated a possible increase trend of the strength of the association of CSBP with CKD with age. With two blood pressure variables jointly entering the multivariable models, SBP, DBP and PP were all not the significant independent predictor of CKD in the 55-64 year age group. In men, with CSBP and SBP jointly entering the multivariable model, SBP was the significant independent risk factor of CKD in each age group, the association of CSBP with CKD was of statistical significance only in 45-54 year age group; with CSBP and DBP jointly entering the multivariable model, CSBP and DBP were both the significant independent risk factors of CKD in 45-54 year age group and 55-64 year age group, in 35-44 year age group, the association of DBP with CKD was of statistical significance while the association of CSBP with CKD was of no statistical significance; with 


\section{Kidney Bloód Pressure Research}

CSBP and PP jointly entering the multivariable model, CSBP was the significant independent risk factor of CKD in all age groups while the association of PP with CKD was of no statistical significance in all age groups. The multicollinearity diagnosis revealed that age, BMI, GLU and TC had a value of variance inflation greater than 10 . After excluding these variables, the multicollinearity was eliminated, as all variance inflation factor values were less than 4 . The results were similar to the models that included these variables.

\section{Discussion}

This large community-based cross-sectional study demonstrated that the risk of CKD tended to rise with CSBP, SBP, DBP and PP in both genders and all age groups. In women CSBP was a better predictor of CKD than SBP, DBP and PP, especially in the elderly; in men CSBP has stronger association with CKD than PP, but not than SBP and DBP. The data also indicated that among the two CKD indicators eGFR and ACR, ACR tended to correlate more closely with the blood pressure measurements.

Table 4. OR and 95\% CI of different blood pressure measurements to identify CKD with CSBP and another blood pressure variable entering into the multivariable logistic models jointly. Variables adjusted in the multivariable logistic models included age, smoking, body mass index (BMI), total cholesterol, fasting glucose, triglyceride, high-sensitivity C reaction protein (hsCRP), history of kidney disease, history of stroke, family history of cardiovascular disease, education level, north or south regions were performed with CSBP and another blood pressure variable entering into the models jointly. The blood pressure measurements were standardized with Z score. Abbreviations: OR, Odds ratio; CI, confidence interval; CKD, chronic kidney disease; CSBP, central aortic systolic blood pressure; SBP, systolic blood pressure; DBP, diastolic blood pressure; PP, pulse pressure

\begin{tabular}{|c|c|c|c|c|c|}
\hline & & \multicolumn{4}{|c|}{ Age groups(yrs) } \\
\hline & & $35-44$ & $45-54$ & $55-64$ & Total \\
\hline \multicolumn{6}{|l|}{ Men } \\
\hline \multicolumn{6}{|l|}{ Model 1} \\
\hline & CSBP Z-score & $0.98(0.71-1.36)$ & $1.30(1.03-1.65)$ & $1.24(0.98-1.55)$ & $1.20(1.03-1.39)$ \\
\hline & SBP Z-score & $1.69(1.24-2.31)$ & $1.39(1.10-1.76)$ & $1.45(1.16-1.82)$ & $1.48(1.28-1.72)$ \\
\hline \multicolumn{6}{|l|}{ Model 2} \\
\hline & CSBP Z-score & $0.99(0.76-1.30)$ & $1.29(1.06-1.56)$ & $1.44(1.21-1.72)$ & $1.30(1.15-1.46)$ \\
\hline & DBP Z-score & $1.85(1.42-2.42)$ & $1.57(1.29-1.91)$ & $1.21(1.01-1.45)$ & $1.45(1.29-1.63)$ \\
\hline \multicolumn{6}{|l|}{ Model 3} \\
\hline & CSBP Z-score & $1.52(1.20-1.93)$ & $1.78(1.49-2.13)$ & $1.41(1.18-1.70)$ & $1.58(1.41-1.77)$ \\
\hline & PP Z-score & $0.93(0.74-1.18)$ & $0.93(0.78-1.11)$ & $1.23(1.02-1.47)$ & $1.04(0.93-1.17)$ \\
\hline \multicolumn{6}{|l|}{$\begin{array}{l}\text { Women } \\
\text { Model } 1\end{array}$} \\
\hline & CSBP Z-score & $1.23(0.95-1.61)$ & $1.50(1.22-1.84)$ & $1.78(1.46-2.16)$ & $1.57(1.39-1.79)$ \\
\hline & SBP Z-score & $1.45(1.11-1.89)$ & $1.27(1.03-1.56)$ & $1.08(0.89-1.31)$ & $1.22(1.07-1.38)$ \\
\hline \multicolumn{6}{|l|}{ Model 2} \\
\hline & CSBP Z-score & $1.48(1.19-1.83)$ & $1.57(1.33-1.86)$ & $1.77(1.53-2.05)$ & $1.68(1.52-1.84)$ \\
\hline & DBP Z-score & $1.20(0.97-1.48)$ & $1.24(1.05-1.46)$ & $1.11(0.96-1.29)$ & $1.15(1.04-1.27)$ \\
\hline \multicolumn{6}{|l|}{ Model 3} \\
\hline & CSBP Z-score & $1.51(1.23-1.84)$ & $1.75(1.48-2.06)$ & $1.90(1.61-2.23)$ & $1.75(1.58-1.94)$ \\
\hline & PP Z-score & $1.18(0.97-1.43)$ & $1.04(0.89-1.23)$ & $0.98(0.84-1.15)$ & $1.06(0.96-1.17)$ \\
\hline
\end{tabular}




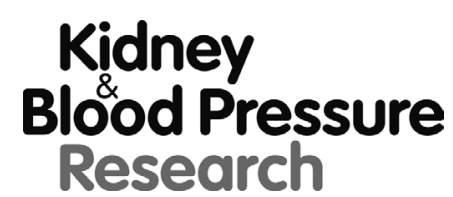

Kidney Blood Press Res 2018;43:1375-1387

\begin{tabular}{l|l}
\hline DOI: $10.1159 / 000492952$ & (C) 2018 The Author(s). Published by S. Karger AG, Basel
\end{tabular}

Published onlıne: 27 August, 2018

www.karger.com/kb

Central hemodynamic measures such as central systolic pressure, central pulse pressure, augmentation index (AI), etc were thought to be a better predicator of kidney dysfunction or damage. The Takenaka et al.'s study found that AI is a risk factor of progression of nondiabetic CKD [22]. Wang's study demonstrated a stronger relation of central blood pressures than peripheral blood pressures with eGFR [23]. Our study showed that CSBP had stronger association with CKD than the brachial blood pressure measurements in women, which supports the perspective above.

Another phenomenon we found was that the superiority of central systolic blood pressure over brachial blood pressures in the prediction of CKD was sex-dependent. In men, CSBP has stronger association with CKD than PP, but not than SBP and DBP. The reason may be that in women there was a greater age-related increase in proximal aortic stiffness due to the unfavorable effects of a lack of estrogen due to menopause [24]. The weaker relationship of CSBP with CKD in the younger age group and stronger association of CSBP with CKD in the older age group in women may provide support to this hypothesis (Table 4). Another potential reason could be the fact that women have more wave reflection due to a shorter body height. In line with this, in Table 1, it is noticeable that despite the lower brachial systolic and diastolic blood pressure in women, their central systolic blood pressure was similar to that in men, and peripheral pulse pressure was actually higher in the former. Our results are also in concordance with the study conducted by Jankowski, who found ascending aortic blood pressure waveform may be related to the risk of coronary disease in women, but not in men [25].

Our data also indicated that among the two CKD indicators eGFR and ACR, ACR tended to correlate more closely with the blood pressure measurements. On the other hand, in the participants diagnosed as CKD in this study, 34 participants had eGFR $<60 \mathrm{~mL} / \mathrm{min} / 1.73 \mathrm{~m}^{2}$ and 837 participants had ACR $\geq 30 \mathrm{mg} / \mathrm{g}$ in women. Therefore, our findings were more a reflection of the association of blood pressure measurements with albuminuria. The results are similar to other studies [8,9]. The Framingham Heart Study reported that carotid femoral pulse wave velocity associated with both urinary ACR and macroalbuminuria but not with CKD (defined by eGFR $<60 \mathrm{ml} / \mathrm{min} / 1.73 \mathrm{~m}^{2}$ ). Temmar et al. reported a more obvious relationship with the pulse pressure measured in the ascending aorta and at the level of renal arteries for proteinuria than for plasma creatinine in the high coronary risk individuals [9]. The underling mechanism of the phenomenon is difficult to interpret. Although there are evidences that increasing blood pressure may impair the renal autoregulation and lead to kidney damage $[26,27]$, urinary albumin excretion has been found to be not only an indication of renal disease, but a risk-marker that reflects generalized vascular damage [28]. Therefore, further studies are warranted to explore the exact mechanism.

Our study has several strengths. The study was community-based and the research population was sampled in consideration of the geographical distribution, socioeconomic level, although it was not a random sample of the Chinese population, it well represented the southern and northern provinces as well as the urban and rural populations in China. The prevalence of current smoking was $56.4 \%$ in men and $6.8 \%$ in women in this study. In the 2015 China adults tobacco survey, the prevalence of the smoking was $52.1 \%$ in men and $2.7 \%$ in women aged 15 years and older [29]. Although the age range in our study is different from that of the national survey in 2015, the prevalence and gender difference are similar. Moreover, the large sample size gave us adequate power to detect modest associations among different gender and age groups. To the best of our knowledge, it is the first study to compare CSBP with brachial blood pressure measurements in relation to the CKD in different gender and age group individuals. In our study, our data demonstrated that CSBP added predictive utility in a model that already considered brachial blood pressure measurements, which is deemed as the standard for introduction of a new biomarker [30]. This study also has several limitations. Firstly, the cross-sectional study design cannot establish a casual relationship between CBP and CKD, further studies will be valuable for confirming these results and findings. Secondly, the CSBP measured in this study is the blood pressure in the 


\section{Kidney \\ Blood Pressure Research}

Zhang et al.: Central Aortic Systolic Blood Pressure and Chronic Kidney Disease Risk

ascending aorta, Hope et al. reported that the aortic systolic pressure was approximately 10 mmHg higher at the level of kidney than in the ascending aorta [31], further study based on the aorta pressure at the level of kidneys may be need. Thirdly, our study only determined serum creatinine and urinary creatinine and microalbumin at one single time-point, which might lead to misclassification of CKD.

\section{Conclusion}

This study compared CSBP with brachial blood pressure measurements in predicting CKD risk in a large scale Chinese middle-aged population. It has been shown that CSBP and brachial BPs are all predictors of CKD in both men and women, and that measurement of CSBP may offer advantages over brachial blood pressure measurements in CKD risk prediction in women, especially in the elderly. Further studies are needed to verify the results and to clarify the underlying mechanism.

\section{Acknowledgements}

The authors acknowledge the contributions of the principal investigators and subcenters. The principal investigators and sub-centers are as follows: Cardiovascular Institute \& Fu Wai Hospital: Zengwu Wang, Linfeng Zhang, Zuo Chen, Xin Wang, Min Guo, Lan Shao, Manlu Zhu, Wen Wang, and Weiwei Chen; Deyang Institute of Hygiene, Sichuan: Guogang Zhang, Anbing Wang, and Xiaoyang Liao; Guangdong Provincial Hospital: Xiaoqing Liu, Jinzhuang Mai, Jiyan Chen, and Meiling Zheng; Hanzhong Hospital, Shanxi: Ruihai Yang and Jun Yang; Jintan Institute of Hygiene, Jiangsu: Jiantao Guo, Xin Zhou, and Xiaoping Yang; Yuxian Hospital, Shanxi: Dongshuang Guo and Shengying Liang; Zhoushan Cardiovascular Institute, Zhejiang: Liansheng Ruan and Chengguo Liu; First Affiliated Hospital of Harbin Medical University, HeiLongjiang: Weimin Li, Yujuan Zhao, and Lihang Dong; Beijing Chaoyang District Center for Disease Control and Prevention, Beijing: Xiaoyan Han; Yunnan center for disease prevention and Control, Yunnan: Yize Xiao; Baotou Medical College, inner Mongolia Autonomous Region: Ying Wang and Chengyi Li; Tibet autonomous region center for disease control and prevention, Tibet: Hui Xiong, Cangjue Gama, Ruodeng Xirao, and Guoxia Bai; Xinjiang Uygur Autonomous Region Yining Center for Disease Control and Prevention, Xinjiang Uygur Autonomous Region: Dongsheng Wang.

This work was supported by the National health and Family Planning Commission of the Peoples' Republic of China (200902001, 201402002). The authors have also received support from Omron Corporation, Kyoto, Japan, for body fat and weight measurement (V-body HBF359); Changsha Aican Electronic Co., Ltd, China, for collecting digital information of ECG (AIKD-B12 V3.0); and HealthSTATS, Singapore, for central blood pressure measurement (Bpro device with A-Pulse CASP software).

The datasets used and/or analyzed during the current study are available from the corresponding author on reasonable request.

\section{Disclosure Statement}

The authors declare no conflict of interests. 


\section{Kidney \\ Blood Pressure Research}

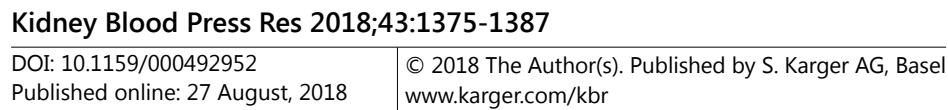

Zhang et al.: Central Aortic Systolic Blood Pressure and Chronic Kidney Disease Risk

\section{References}

1 Kintscher U: The burden of hypertension. EuroIntervention 2013;9:R12-15.

2 Viera AJ, Hawes EM: Management of mild hypertension in adults. BMJ 2016;355:i5719.

-3 Remington JW, Wood EH: Formation of peripheral pulse contour in man. J Appl Physiol 1956;9:433-442.

$\checkmark 4$ Cohen DL, Townsend RR: Central blood pressure and chronic kidney disease progression. Int J Nephrol 2011;2011:407801.

5 Roman MJ, Devereux RB, Kizer JR, Lee ET, Galloway JM, Ali T, Umans JG, Howard BV: Central pressure more strongly relates to vascular disease and outcome than does brachial pressure: the Strong Heart Study. Hypertension 2007;50:197-203.

-6 Pini R, Cavallini MC, Palmieri V, Marchionni N, Di Bari M, Devereux RB, Masotti G, Roman MJ: Central but not brachial blood pressure predicts cardiovascular events in an unselected geriatric population: the ICARe Dicomano Study. J Am Coll Cardiol 2008;51:2432-2439.

7 Williams B, Lacy PS, Thom SM, Cruickshank K, Stanton A, Collier D, Hughes AD, Thurston H, O'Rourke M, CAFE Investigators; Anglo-Scandinavian Cardiac Outcomes Trial Investigators; CAFE Steering Committee and Writing Committee: Differential impact of blood pressure-lowering drugs on central aortic pressure and clinical outcomes: principal results of the Conduit Artery Function Evaluation (CAFE) study. Circulation 2006;113:1213-1225.

8 Upadhyay A, Hwang SJ, Mitchell GF, Vasan RS, Vita JA, Stantchev PI, Meigs JB, Larson MG, Levy D, Benjamin EJ, Fox CS: Arterial stiffness in mild-to-moderate CKD. J Am Soc Nephrol 2009;20:2044-2053.

-9 Temmar M, Jankowski P, Peltier M, Mouquet V, Debicka-Dabrowska D, Hamida F, Kawecka-Jaszcz K, Safar ME: Intraaortic pulse pressure amplification in subjects at high coronary risk. Hypertension 2010;55:327332.

10 Hao G, Wang Z, Zhang L, Chen Z, Wang X, Guo M, Tian Y, Shao L, Zhu M: Prevalence of microalbuminuria among middle-aged population of China: a multiple center cardiovascular epidemiological study. Angiology 2015;66:49-56.

11 Wang Z, Zeng X, Chen Z, Wang X, Zhang L, Zhu M, Yi D: Association of visceral and total body fat with hypertension and prehypertension in a middle-aged Chinese population. J Hypertens. 2015;33:1555-1562.

12 Wang Z, Hao G, Zhang L, Chen Z, Wang X, Guo M, Tian Y, Shao L, Zhu M: Central systolic blood pressure is associated with ethnicity and cardiovascular disease risk factors in Chinese middle-aged population. Eur J Prev Cardiol 2016;23:228-236.

13 Hao G, Wang Z, Zhang L, Chen Z, Wang X, Guo M, Tian Y, Shao L, Zhu M: Thresholds of Central Systolic Blood Pressure in a Normotensive Chinese Middle-Aged Population. Angiology 2016;67:174-179.

14 People's Republic of China-United States Cardiovascular and Cardiopulmonary Epidemiology Research Group: An epidemiological study of cardiovascular and cardiopulmonary disease risk fators in four populations in the People's Republic of China: baseline report from the P.R.C.-U.S.A. collaborative study. Circulation 1992;85:1083-1096.

15 Zhou BF, Zhang HY, Wu YF, Li Y, Yang J, Zhao LC, Zhang XH: Ecological analysis of the association between incidence and risk factors of coronary heart disease and stroke in Chinese populations. CVD Prevent 1998;1:207-216.

16 Garcia-Ortiz L, Recio-Rodríguez JI, Canales-Reina JJ, Cabrejas-Sánchez A, Gomez-Arranz A, Magdalena-Belio JF, Guenaga-Saenz N, Agudo-Conde C, Gomez-Marcos MA, EVIDENT Group: Comparison of two measuring instruments, B-pro and SphygmoCor system as reference, to evaluate central systolic blood pressure and radial augmentation index. Hypertens Res 2012;35:617-623.

17 Ott C, Haetinger S, Schneider MP, Pauschinger M, Schmieder RE: Comparison of two noninvasive devices for measurement of central systolic blood pressure with invasive measurement during cardiac catheterization. J Clin Hypertens (Greenwich) 2012;14:575-579.

18 Hao G, Wang Z, Zhang L, Chen Z, Wang X, Guo M, Tian Y, Shao L, Zhu M: Thresholds of Central Systolic Blood Pressure in a Normotensive Chinese Middle-Aged Population. Angiology 2016;67:174-179.

19 Williams B, Lacy PS, Yan P, Hwee CN, Liang C, Ting CM: Development and validation of a novel method to derive central aortic systolic pressure from the radial pressure waveform using an n-point moving average method. J Am Coll Cardiol 2011;57:951-961. 


\section{Kidney \\ Blood Pressure Research}

20 Levey AS, Stevens LA, Schmid CH, Zhang YL, Castro AF 3rd, Feldman HI, Kusek JW, Eggers P, Van Lente F, Greene T, Coresh J; CKD-EPI (Chronic Kidney Disease Epidemiology Collaboration): A new equation to estimate glomerular filtration rate. Ann Intern Med 2009;150:604-612.

-21 Stevens PE, Levin A, Kidney Disease: Improving Global Outcomes Chronic Kidney Disease Guideline Development Work Group Members: Evaluation and management of chronic kidney disease: synopsis of the kidney disease: improving global outcomes 2012 clinical practice guideline. Ann Intern Med 2013;158:825-830.

-22 Takenaka T, Mimura T, Kanno Y, Suzuki H: Qualification of arterial stiffness as a risk factor to the progression of chronic kidney diseases. Am J Nephrol 2005;25:417-424.

23 Wang KL, Cheng HM, Chuang SY, Spurgeon HA, Ting CT, Lakatta EG, Yin FC, Chou P, Chen CH: Central or peripheral systolic or pulse pressure: which best relates to target organs and future mortality? J Hypertens 2009;27:461-467.

24 Waddell TK, Dart AM, Gatzka CD, Cameron JD, Kingwell BA: Women exhibit a greater age-related increase in proximal aortic stiffness than men. J Hypertens 2001;19:2205-2212.

25 Jankowski P, Kawecka-Jaszcz K, Czarnecka D, Bryniarski L: Ascending aortic blood pressure waveform may be related to the risk of coronary artery disease in women, but not in men. J Hum Hypertens 2004;18:643648.

26 Hill GS, Heudes D, Jacquot C, Gauthier E, Bariéty J: Morphometric evidence for impairment of renal autoregulation in advanced essential hypertension. Kidney Int 2006;69:823-831.

-27 Hashimoto J, Ito S: Central pulse pressure and aortic stiffness determine renal hemodynamics: pathophysiological implication for microalbuminuria in hypertension. Hypertension 2011;58:839-846.

-28 Deckert T, Feldt-Rasmussen B, Borch-Johnsen K, Jensen T, Kofoed-Enevoldsen A: Albuminuria reflects widespread vascular damage. The Steno hypothesis. Diabetologia 1989;32:219-226.

29 Yan Y, Nan Y, Tu MW, Wang JJ, Wang LL, Jiang Y: Major finding of 2015 China adults tobacco survey. Chin J Health Manage 2016;10:85-87.

30 Hlatky MA, Greenland P, Arnett DK, Ballantyne CM, Criqui MH, Elkind MS, Go AS, Harrell FE Jr, Hong Y, Howard BV, Howard VJ, Hsue PY, Kramer CM, McConnell JP, Normand SL, O’Donnell CJ, Smith SC Jr, Wilson PW, American Heart Association Expert Panel on Subclinical Atherosclerotic Diseases and Emerging Risk Factors and the Stroke Council: Criteria for evaluation of novel markers of cardiovascular risk: a scientific statement from the American Heart Association. Circulation 2009;119:2408-2416.

-31 Hope SA, Tay DB, Meredith IT, Cameron JD: Waveform dispersion, not reflection,may be the major determinant of aortic pressure wave morphology. Am J Physiol Heart Circ Physiol 2005;289:H2497-2502. 